\title{
The "Ins and Outs" of APCs: An Overview of Automatic Passenger Counters
}

\author{
Michael R. Baltes \\ Joel R. Rey \\ University of South Florida, Tampa
}

\begin{abstract}
Experience has shown that manual data collection via ridechecks is one of the most costeffective data collection methods for most transit systems when evaluating ridership levels and system performance. Unfortunately, this manual method of collecting ridership data and system operational information produces both limited system wide and route specific reports due to the amount of manual ridechecking required and the manual data processing involved. This is especially true for langer transit systems. One possible alternate solution for a transit system to meet its need for reliable, accurate, detailed, up-to-date, and cost-effectively-obtained ridership data is through the use of automatic passenger counters, or APCs. Unlike manual ridechecks, anAPC system provides a transit system with an automated method for collecting information on the number of passenger boardings and alightings at a variety of system levels including route, route segment, or specific bus stops by time of day and by day of week, for example. This paper provides a general overview of APC systems. Included is a discussion of APC components, a review of typical transit system data needs, a list of current worldwide APC vendors, the results of a survey of North American transit systems related to APC usage, and a summarization of important issues for transit systems to consider when contemplating integrating APC systems on board their vehicles.
\end{abstract}




\section{Introduction}

Efficient and effective public transit service delivery depends on the interplay of a multitude of internal and external factors, but in large part depends on accurate information collected about transit ridership and overall transit system operations. When provided with information about the transit system, the system's overall performance and performance of individual routes and route segments can be assessed with great accuracy by system planning, scheduling, and management staff. Accurate and comprehensive ridership data will indicate areas of strength and weakness in transit operations and will support and justify the corrective efforts established by transit agency staff.

Historically, the data most appropriate for examining the performance of a transit system have been obtained from manually-derived passenger ridechecks (a ridecheck is a ride, usually on a transit bus, on which staff manually tabulates the number of passenger boardings and alightings at each stop by time of day, tracks ontime performance, and observes the operator's skills and compliance with rules and standards). Generally, these ridechecks reveal peak passenger loads by bus stop and run times on a specified route. The ridechecks also show the demand for the service at the time, location, and direction of that demand using these data. Frequency and running time decisions can be made so that the supply of service corresponds precisely with the actual demand for that service. This will provide optimal economies to the transit system and, hopefully, improve the provision of transit service to the system's customers.

Experience has shown that many transit systems use labor-intensive manual ridechecks during specific service periods to collect ridership (number of boardings and alightings at the stop level) and operational data such as run times. Currently, this is one of the most cost-effective data collection methods for most transit systems. Unfortunately, this manual method of collecting ridership data and system operational information produces both limited systemwide and route specific reports due to the amount of manual ridechecking required and the manual data processing involved. This is not to say, however, that processing this limited amount of manually-collected information into reports does not provide beneficial information to 
support decisions by transit system staff. Nevertheless, the limited information obtained associated with collecting and processing information into a comprehensive database by manual ridechecks has persuaded some transit systems to pursue the utilization of an automated alternative data collection method.

One possible alternate solution for a transit system to meet its need for reliable, accurate, detailed, up-to-date, and cost-effectively-obtained ridership data is through the use of automatic passenger counters, or APCs. Unlike manual ridechecks, APCs provide a transit system with an automated method for collecting the number of passenger boardings and alightings at a variety of system levels including route, route segment, or specific bus stops by time of day and by day of week, for example. Unlike the limited information available via manual ridechecks, APCs provide the transit agency the opportunity to automatically collect a host of additional information about operations, as well. In addition, APCs also enable transit systems to automatically upload the passenger and run time data that were collected through various electronic means, thus eliminating the errors that can accompany manual data entry.

\section{What is an APC System?}

As stated above, an APC system provides an automated method for collecting passenger and system data. Among the additional parameters that can be measured by an APC system are maximum and minimum load points, boarding and alighting rates, vehicle dwell times, door cycles, wheelchair lift cycles, distance traveled, and vehicle average speed.

An APC system can be seen as akin to a microscope in that it permits transit system staff to intently focus on the productivity and quality of their system's performance at various levels, ranging from individual bus stops or route segments, to time point segments, and to the overall transit system as a whole. Just as the development of new technologies such as the Internet has increased knowledge about the world around us, an APC system will allow transit staff to identify problems that may not have been possible to identify when analyzing data gathered via manual ridechecks and to create innovative strategies for improving their transit system's performance. 


\section{Basic Components}

To collect ridership data such as load levels and their locations, a number of APC hardware components are required including:

- counting sensors (treadle mats, I-R beams, etc.);

- an odometer sensor for location referencing;

- a clock to determine the time that the passenger activity occurred (usually in the microprocessor);

- a data processing unit to tabulate, accumulate, and store passenger activity data onboard the bus;

- data storage devices consisting of either integrated circuit semiconductor memory; cassette tape; or 51/4-, 31/2-inch computer diskettes, or hard drive (all three usually contained in the data processing unit);

- a power supply to convert primary bus voltage (usually 12 or 24 volts DC nominal) to the APC system;

- engine sensors to register engine dwell and idle times;

- wheelchair lift sensors to register wheelchair lift activity;

- door sensors to register door openings and closings; and

- either radio signposts or some type of global positioning system (GPS) technology to improve the confidence in the location referencing of odometer readings.

The current state-of-the-art methods of automatic passenger counting currently in use can be divided into several technologies: infra-red (I-R) beams, treadle mats, optical sensors, and low ultrasonic frequency sensors. (These latter two technologies have specific application on low-floor buses, which present a special problem for APC I-R beam and treadle mat technologies when counting passenger activity due to the width, height, and design of the buses' front door. However, at the time of this writing, these two technologies were not being widely used in North American transit systems. For this reason, in-depth information about these two technologies has not been included herein.) To illustrate, the APC technology that utilizes I-R beams computes the total number of boardings and alightings by tabulating the number of times the beam is "interrupted" by a passenger entering or exiting the bus. Generally, 
the I-R beams are placed at the waist height of passengers. Algorithms are specifically built into the APC's proprietary software to take into account the under- and over-counting of passengers created by multiple passengers crossing the beam simultaneously and passengers exiting through the front door on a two-or-more-door bus (accomplished through the sequence in which the I-R beams are broken).

APC treadle mat technology tracks passenger boardings and alightings through passenger foot pressure on sensing contacts/switches positioned in the "tread" of the bus's stairwell steps. The APC's treadle mats are designed to replace the bus's existing stairwell mats. Proper fit of the treadle mats is critical for safety reasons and to properly determine the stream of passenger activity in each stairwell. Similar to the APCs that utilize I-R beams, the APC's software is tailored to account for the underand over-counting of passengers.

Optical sensors are a non-contact vision system that optically detects passenger activity. This specific APC technology was designed for application on low-floor buses. A single overhead optical sensor is mounted in each doorway of the vehicle to detect passenger boardings and alightings. One of the strengths of this type of system over APCs that use treadle mats or I-R beams is its ability to detect multiple passengers in doorways and simultaneous bi-directional passenger flows (particularly useful on vehicles with only one door).

Ultrasonic frequency sensors are acoustic in nature and track passengers by transmitting and receiving ultrasonic pulses, i.e., pulses of sound that are above the range of human hearing (typically 30 to $100 \mathrm{kHz}$ ). At the time of the project upon which this paper is based, ultrasonic frequency sensor technology for specific application on low-floor buses was still in the beta test stages. Therefore, specific information about this APC technology could not be included herein. Currently, however, this technology is being tested at OC Transpo in Ottawa-Carleton, Canada.

For each of the APC technologies, the location of the bus when the APC system is performing actual counts of passenger boardings and alightings while in revenue service is inferred by using an odometer. Confidence in the position of the bus is improved by using either electronic signposts or GPS. Signpost technology has been in use for many years by a number of transit agencies in conjunction with APC 
systems and other technologies such as automatic vehicle location (AVL), but GPS is a relatively new technology that is now being more widely incorporated with APCs and other technologies such as AVL to greatly improve locational accuracy of transit vehicles. Signposts are commonly ground-based, short-range radio transmitters located throughout a given transit system's service area that provide a number of accurately known locational references. Between signposts, the bus's location is deduced by the APC's data processing unit by correlating the distance traveled provided by the odometer with the route the vehicle is traveling while in revenue service.

Unlike the ground-based signpost technology used with some APC systems, APCs that utilize GPS technology make use of satellites positioned around the earth to determine, with great accuracy (usually within a few meters), the location of the transit buses that are affixed with GPS receivers. The GPS satellites transmit signals that permit, with tremendous accuracy, the identification of the global position of the GPS receiver(s) placed on the transit buses, therefore also allowing for the precise determination of the location of the transit buses within the transit agency's service area. Specifically, the GPS receiver(s) placed on the transit buses use the GPS satellites as reference points for triangulating a precise position on earth. Two satellite measurements determine an intersection of two spheres and a third measurement identifies two points common to the spheres, while a fourth measurement determines the specific point. One point that should be noted is that, despite the improved accuracy of location determination using AVL/GPS technologies, it is still possible for location errors to occur, e.g., weakened signal or complete signal loss resulting in positional inaccuracies of, or total loss of positional information for, specific time point/bus stop location(s).

To clarify the workings of an APC system for the reader, a simple flow diagram contained in Figure 1 illustrates a hypothetical APC system. It should be pointed out that APC systems (both hardware and software) currently in use at transit properties throughout North America have been customized a great deal to fit the particular data needs of these transit agencies as well as the design problems related to the installation of an APC system that are created by a transit agency's bus fleet being composed of different bus types from a variety of manufacturers. Therefore, no two 
APC systems in operation are identical and, also, for these reasons, an "off-the-shelf" APC system does not truly exist that can be easily integrated into and satisfy the diverse needs of a particular transit system. It is also for these reasons that detailed information related to the per unit cost of APCs could not be obtained and included.

Once the boarding and alighting data are collected by an APC system, they can either be stored in integrated circuit semiconductor memory, on a cassette tape, or on a $5 \frac{1}{4-\text { or }} 3 \frac{1}{2}$-inch computer diskette. The current methods for data transfer from the APC's data processing unit consist of the following:

- Transit agency staff manually retrieves collected data from either a cassette tape, diskette (51/4- or 31/2-inch), or magnetic data card (other forms of storage media may be available).

- Transit agency staff manually retrieves the collected data by linking to the APC's data processing unit via a portable laptop computer which transfers the data to a diskette or hard drive in the laptop.

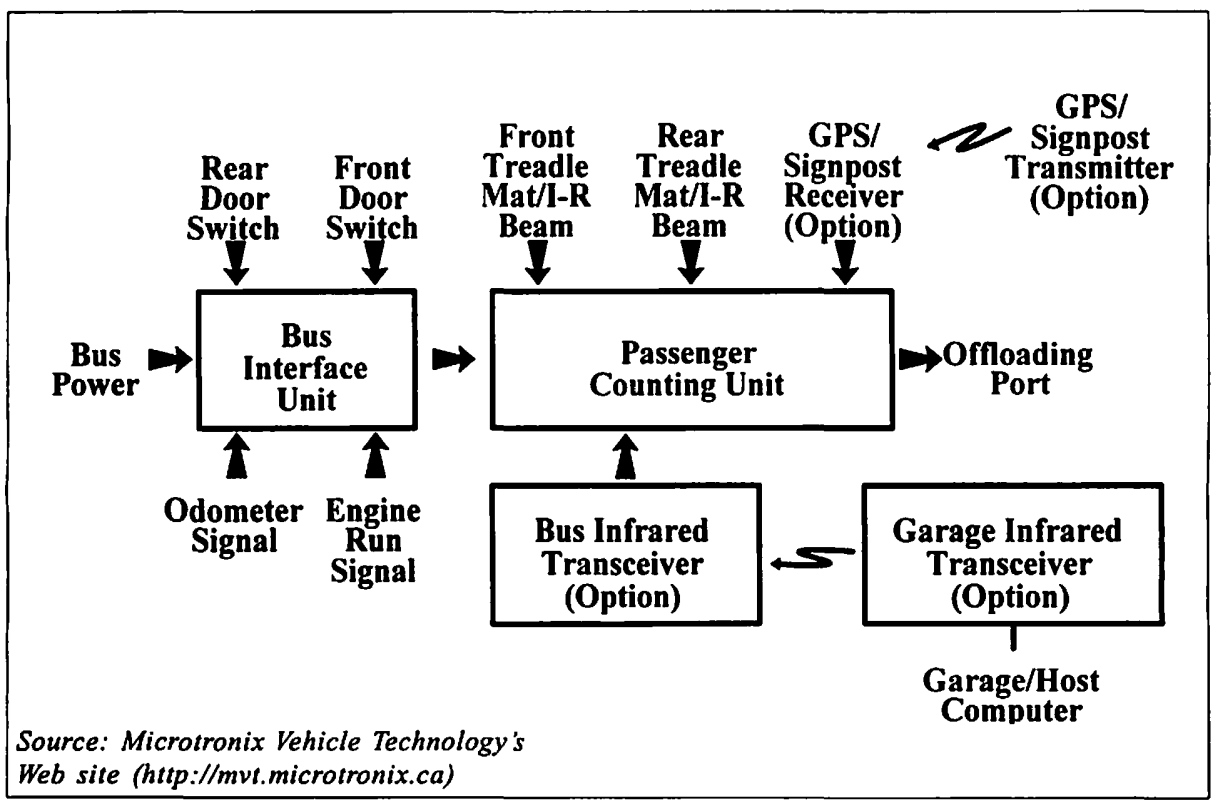

Figure 1. Illustration of a hypothetical APC system and related components. 
- Transit agency staff manually links, usually using a telephone line, the APC's data processing unit to a wayside computer located at the transit system's garage, for example.

- Equipment is used to automatically transfer data from the APC's data processing unit via the vehicle radio or a communication system such as AVL to a central computer located at the transit system's garage or central facility. The ridership data can be transmitted in real time with the vehicle location provided by the AVL system.

- An optical link (I-R beam) to automatically transfer data from the APC's data processing unit to a wayside computer at the transit system's garage, for example.

In addition to the basic APC components listed previously, a transit system that is evaluating the potential use of APCs will also have to consider additional equipment for the storage and processing of the resulting APC obtained data. This equipment may include computer equipment (laptops, PCs, mainframe, LAN), long-term storage devices, and supplemental analysis software such as SPSS or SAS.

\section{Typical Data Needs}

For a transit system to effectively and objectively evaluate its performance and identify potential areas for service improvement, a significant amount of information about passenger boardings and alightings is required at both the micro- and macro-level. Collecting and evaluating this information is critical for a transit system to make effective decisions about changes in service provision that will translate into increased operating efficiencies and improve the provision of transit service to the general public. In deciding whether or not to implement an APC system, a transit system first needs to evaluate its data needs and the intended use(s) of the data by its service planning and scheduling departments once it has been collected by the APC system. This is a critical first step since the data an APC system gathers can be tailored to meet the specific data and report generation requirements specified by a transit system. 
Typical data needs can be grouped into several general categories as follows:

- Data for Service Planning - data related to the daily variation in total passengers, boarding and alighting distributions, county of operation, overcrowding(standees) times/locations, and actual run times compared to scheduled times by route and route segment, by time period and day of week. From these data, a transit system can monitor the minimum and maximum passenger loads by direction, determine average speeds for the purpose of restructuring routes and headways, and derive various other measures for comparison with its route- and system-level service standards. In addition, these data can also be used to discover if there are any seasonal variations in ridership and run times. The need here is to conduct frequent enough data collection so that the statistical significance of the daily variations can be identified with a high degree of confidence.

- Data for Scheduling - data related to individual stop and trip boardings and alightings, run times, dwell times, deadheading times, time point arrival and departure times, and on-time performance. From these data, for example, run time analyses can be performed to monitor and adjust trip cycle times to maximize both cost efficiencies and passenger comfort.

- Data for Transportation Management-data related to the daily exceptions to the scheduled time point and stop arrivals and departure times of buses, passenger loads at important locations, and compliance with National Transit Database (NTD, formerly Section 15) reporting requirements.

\section{APC Studies}

To identify current information on APC technologies, an in-depth review of available APC literature was conducted, as was an Internet search using key words and phrases such as "automated passenger counting systems" and "vehicle technology." Unfortunately, the APC literature available for review is sparse and is not as current as originally thought. However, documentation from several APC demonstration projects conducted by transit systems were identified and reviewed. 
One of the primary concerns about the data that are obtained from an APC system is the accuracy of the passenger boardings and alightings. Overall, the APC information indicates that, for routes with higher volumes of passenger activity, APCs have a tendency to undercount rather than overcount passenger activity. Regarding specific activity, the literature points out that passenger boardings tend to be counted more accurately than alightings. The most likely explanation for this is that passengers boarding a transit vehicle tend to do so in a more orderly fashion (i.e., one at a time in a queue) than when alighting, particularly on buses with two or more rear doors. When evaluating APCs, transit agencies that use manual data collection methods must weigh this problem of APCs under- and over-counting passengers in certain situations against the errors that can typically occur during manual data entry of the ridecheck collected data. However, it should further be noted that the problem of under- and over-counting passengers can also occur during manual data collection, especially during heavy passenger loads.

In 1991, the Washington Metropolitan Area Transit Authority (WMATA) conducted a six-week test of APC systems to determine their feasibility for collecting data for the conduct of economic route analyses (WMATA 1991). The route selected for the APC test represented a typical bus route in the WMATA service area. Three buses were equipped with APC systems using I-R beams. In addition to the on-bus APC systems, nine radio signposts were installed as well to improve the location accuracy of the passenger counting. During peak hours, the route offered six-minute headways, requiring 13 buses. At the time the tests were conducted, weekday ridership was about 7,700 passengers. The accuracy of the APC-collected data was measured at the route, route segment, and bus stop level. Manual ridechecks were performed with persons on the APC-equipped buses simultaneously as the APCs recorded passenger activity.

During the WMATA tests, the ridecheckers recorded more than 17,000 boardings and an almost equal number of alightings. On average, the boardings recorded by the APC systems varied by less than one percent (about 99 percent accurate) from the manually-recorded data. With respect to alightings, the average variance between the APCs and manually-recorded data was less than two percent (about 98 percent 
accurate). In addition, maximum load data between the APC systems and manuallycollected data compared favorably. Overall, the tests concluded that the APC systems performed equal to that of the manual ridecheckers. It should be mentioned that communication with several APC vendors and transit systems that are currently using APC technology determined that the counting accuracy for both boardings and alightings, even during peak loads, is consistently above 90 percent regardless of APC system configuration (i.e., sensor type used, etc.).

A study of APC systems at OC Transpo located in Ottawa-Carleton, Canada, uncovered some of the reasons why APC systems that use I-R beams may inaccurately count passenger activity (OCRTC 1986). Some of the reasons listed include mistakenly counting umbrellas or waving arms of passengers that crossed the I-R beams as stairwell activity, missing very small children who walked under the I-R beams, undercounting groups of passengers who boarded or alighted very close together, counting passengers more than once who swayed back and forth while in the stairwell, mistakenly counting a boarding passenger as alighting or vice versa (particularly problematic on one-door buses), counting errors arising from dirt accumulating on the front door windows which distorted the I-R beam when the door was open, and cold weather adversely affecting the performance of the I-R sensors. These problems were ameliorated by simple routine maintenance such as cleaning the front door windows, heating the I-R beam sensor heads during cold weather, disabling the counting mechanism when the doors are closed, and optimally locating the sensors at the front and rear doors. It should be noted that correction algorithms will still need to be developed and utilized to account for any remaining error(s).

In addition to the problems encountered with I-R beams inaccurately counting passenger activity, treadle mats also have difficulty accurately counting passengers in a variety of situations. These situations usually occur when passengers behave inconsistently when boarding or alighting vehicles. For example, young passengers or commuters rushing to catch the bus will sometimes skip a step in the vehicle's stepwell; confused individuals will sometimes walk partway up the steps to ask a driver for information while stepping on the mat, then back out of the vehicle after a response has been given; and passengers waiting in line to board a vehicle will 
sometimes rock back and forth with a heel-to-toe motion on one or more of the vehicle's steps thereby creating confusion in the APC system as to the direction of travel (boarding vs. alighting) (Microtronix 1997).

In addition, treadle mats are more susceptible to environmental/climatic conditions than I-R beams, according to a demonstration project of APCs at Denver Regional Transportation District (RTD) (Denver RTD 1985). These include excessive vibration caused by poor road conditions which can loosen the mats from the vehicle steps, and the seepage of water and/or salt (especially in winter climates) into the mat(s) when punctures occur due to rocks, grit, glass, etc. However, it should be noted that the RTD study also concluded that I-R beams are susceptible to vibration as well, which can cause misalignment of the beams. In addition, the study noted that the misalignment of the I-R beams can also occur due to vandalism.

In addition to being able to collect transit system data automatically, APCs also offer significant data collection cost savings. In at least one study of the economic feasibility of APCs conducted by WMATA, it was noted that between a 66 and 90 percent cost savings can be realized via the use of APCs over manual ridecheckers.

The review of APC information and an Internet search developed a list of APC vendors, as shown in Table 1. From these sources, a list of 41 transit systems that have in the past used or are currently using APCs in North America was also compiled. A survey of these particular transit systems was conducted to gather information about their experiences with APC systems.

\section{Transit System Survey}

To identify current APC technologies/vendors, a brief 13-question APC-related survey was developed and faxed to 41 transit agencies in the United States and Canada to determine if they have ever used or are currently using APC technology, the type of technology employed (if applicable), the vendor of the technology, uses for the ridership data once collected by the APC system, and the accuracy of the ridership data collected by the APC system, among other information. The survey was sent to the planning director or applicable position or the APC technician, where identified, within each transit agency. Of the 41 transit systems surveyed, nine were Canadian properties. 


\section{Table 1 \\ APC Vendors}

\section{U.S. Vendors}

Urban Transportation Associates (UTA) (I-R Beams, GPS Locator, Proprietary APC Data Analysis Software), 700 East McMillan, Ste. 302, Cincinnati, OH 45206, (T) 513/961-0099, (F) 513/751-2821

\section{Canadian Vendors}

Microtronix Vehicle Technologies (MVT) (Treadle Mat and Optical Sensor, Signpost or GPS Locator), 200 Aberdeen Drive, London, Ontario N5V 4N2, (T) 519/659-9500, (F) 519/659-8500,mvt@microtronix.com

ILI Technologies, Inc. (Treadle Mat \& Low Ultrasonic Frequency, Signpost, Proprietary APC Data Analysis Software), 830, 407 - 2nd Street S.W., Calgary, Alberta T2P 2Y3, (T) 403/291-9161, (F) 403/291-9660, ilitech@telusplanet.net

Wardrop Applied Systems, Inc. (Proprietary APC Data Analysis Software), 6006725 Airport Road, Mississauga, Ontario L4V IV2, (T) 416/673-3788, (F) 416/ 673-8007

Red Pine Instruments, LTD. (I-R Beams, Signpost/GPS Locator, Proprietary APC Data Analysis Software), RR 1, Denigh, Ontario K0H 1L0, (T) 613/3332776, (F) NA

\section{European Vendors}

Dilax AG (information on APC technology available), Seepanorama, $\mathrm{CH}-8559$, Fruthwilen Lankwitzer Strasse 3, D-12209, Switzerland (T) (+41) 716637575 , (F) (+41)7166375 76,dilax@paus.ch

INIT (Passive I-R Detector Array, Signpost or GPS, Proprietary APC Data Analysis Software), Haid-und-Neu-Strasse 7-9, D-7500 Karlsruhe, Berlin, Germany, (T) 0-721-69-10-73-76, (F) 0-721-69-68-08, postmaster@init-ka.de

Note: This table is provided for informational purposes only; it is not intended to be an endorsement of the APC vendors or their equipment. The information contained in the table was collected in March 1999. MVT and Wardrop Applied Systems, Inc. work jointly to provide APC systems. 
A total of 25 surveys was returned, with 14 of the responding transit systems indicating that they currently use or have in the past used APC systems. With regard to the different types of APC technology employed by each, three systems indicated that they use(d) treadle mats, and the rest use either dual, multiple, or passive I-R beam technology. Discussions with staff at several of the responding transit systems revealed the fact that an APC system's counting technology (e.g., treadle mat, I-R beam, etc.) is not as critical to the quality of the passenger and system data collected as is the APC system's software. The transit staff that were interviewed noted that the APC system's software is the most critical link to obtaining quality passenger count and other system data. In addition, the transit system staff specifically noted that there is a nominal difference with regard to accuracy between the differing APC counting technologies. Also, several of the transit system staff commented that signpost technology works better in a highly urban environment, particularly in a metropolitan downtown setting with high-rise buildings, and that, overall, there is only a marginal difference between signpost and GPS technology for use in improving the confidence of an APC system's odometer readings.

On the survey, transit systems were also asked to provide information about their use(s) for the data collected by their APC systems. Responses to the survey show that the majority of the transit systems use APC-collected data primarily to create, evaluate, and adjust schedules and run times, and plan and justify route changes, as shown in Table 2. Other, less common uses of the APC-generated data are for the purpose of NTD reporting requirements, monitoring driver performance, and determining the location of bus stops. Approximately 93 percent of the transit systems that indicated that they use(d) an APC system are satisfied with the system's overall reliability and about the same percent are satisfied with the accuracy of their obtained passenger information; the vast majority of these transit systems noted that they are achieving accuracy levels of 90 percent and above regardless of the APC counting technology employed. Last, of the 14 transit systems that reported that they use(d) an APC system, 12 noted that they periodically verify the accuracy of the information collected by their APC systems via only manual ridechecks while the other two transit systems use a combination of manual ridechecks and GFI fareboxgenerated information. 


\begin{tabular}{|lc|}
\hline \multicolumn{2}{|c|}{ Table 2} \\
Transit System Uses for APC Systems \\
\hline Uses for APC Systems & $\begin{array}{c}\text { Number of } \\
\text { Responding } \\
\text { Transit Systems }\end{array}$ \\
\hline Create/evaluate/adjust run times/schedules & 14 \\
Plan/justify route changes & 13 \\
Evaluate marketing strategies & 3 \\
Estimate expected revenue & 1 \\
Determine fleet needs & 2 \\
Monitor driver performance & 3 \\
Determine location of stop facilities & 5 \\
NTD reporting & 6 \\
Other & 2 \\
\hline
\end{tabular}

\section{Important Considerations}

In sum, there are many aspects to consider when contemplating the implementation of APCs by a transit system. Specifically, a number of important issues were revealed during the course of this research related to integrating APCs into a transit system. These important considerations are as follows.

1. It is critical that scheduling, planning, management, and maintenance staff all embrace their system's APC program in order to ensure that the resulting data will be properly utilized and that the APC units will be properly maintained.

2. For any APC system to perform up to expectation it is critical, in conjunction with other factors, that a transit agency have a very detailed bus stop inventory that includes the number of bus stops and the exact mileage between the bus stops at the individual route level for the entire system, assuming that all routes will be 
sampled using the APC system, prior to APC system installation. This will allow for precise analysis of passenger activity at the bus stop and route segment levels as well as on-time performance for both individual routes and the system as a whole.

3. Agency staff must be prepared to handle and analyze the voluminous amount of information gathered by the APC systems in comparison to the typically scant information obtained from manual ridechecks. This may require additional training of planning and scheduling staff.

4. Agency staff should prepare a detailed sampling plan that specifies exactly how and when the APC-equipped buses will collect passenger data prior to APC system installation. This becomes especially important if the APC-equipped buses will be used to satisfy NTD reporting requirements or for the statistical validity of data collected for other reasons.

5. Environmental conditions in which a transit system operates should be taken into account prior to selecting an APC counting technology since rain, dirt, snow, salt, and extreme fluctuations in temperature may adversely affect the operation of a specific counting technology. For example, treadle mats have a slight tendency for water to seep into the mats which can lead to corrosion of the mat's internal switches, particularly in climates that experience prolonged winters or rainy seasons.

6. Agency staff should consider carefully their particular data needs and the purposes for which they will be utilizing APC-generated information when determining how many vehicles to outfit with APCs. APC systems can be quite costly, especially with custom modifications, and putting units on more vehicles than a system actually requires is an unnecessary waste of funds. The rule of thumb suggested by a number of APC vendors and transit systems currently using APCs is to outfit 10 percent of a system's active vehicle fleet with APC units (since sampling is typically used when collecting ridership data [both manually and automatically], it is not necessary to equip a larger percentage of a system's fleet with APCs).

7. Consideration should also be given to the fleet mix on which the APCs will be installed to make sure that routes requiring buses with smaller turning radii or buses with large seating capacities (articulated) be accommodated. 
8. The portability of the APC units between buses of varying types and the wiring of additional buses to accept APC systems should be another consideration at the time of initial unit installation. This is important since a particular bus may break down while in revenue service for a variety of reasons and it may be necessary to transfer the APC system to another bus of the same type.

9. Agency staff should be familiar with and account for the Transit Communications Interface Protocols (TCIP) family of standards including SAE J1708 (supports communication among devices installed on a transit bus) and J1587 (an extension of J1708 which defines data and message identifiers) prior to APC system installation so that subsequent integration with other advanced technologies for transit (e.g., AVL) will be possible.

10. Care should be taken in the placement of APC counting sensors and central processor units. Treadle mats need to be perfectly fitted on the vehicles steps to ensure that all passenger activity is recorded and that there are no extended mat edges that can create a hazard to boarding/alighting passengers or make the mat more susceptible to damage or vandalism. Similarly $\mathrm{I}-\mathrm{R}$ beam sensors should be as unnoticeable to passengers as possible and completely out of the passenger stream so that inadvertent bumping and/or vandalism will not occur that could possibly effect sensor alignment and, therefore, their counting accuracy. If possible, the APC system central processing unit or other modular components should also be located in a hidden and "out-of-the-way" location to prevent damage and/or vandalism.

Based on these observations, it is apparent that the decision to utilize APCs requires more than just selecting and installing a particular APC technology. Considerable planning needs to occur prior to, during, and after the installation of APCs, even if only for demonstration purposes. Only with this planning and proper preparation can the efficient and effective collection of accurate information about transit ridership and overall transit system operations be realized using APCs.

\section{Acknowledgments}

The authors wish to thank Mark Lowenstine of Microtronix Vehicle Technologies LTD, Steve Gallant of Wardrop Engineering Inc., Tom Kowalski of Urban Trans- 
portation Associates Inc., and Horst Gerland of INIT for sharing their vast knowledge and experiences about APCs. In addition, the authors would like to thank Dennis Hinebaugh and Victoria Perk of CUTR for their editorial comments regarding content.

\section{References}

IBI Group. 1985. Automatic passenger counting demonstration-Evaluation report. Regional Transportation District, Denver.

Microtronix Vehicle Technologies LTD. 1997. Informational memorandum sent to CUTR.

Ottawa-Carleton Regional Transit Commission. 1986.APC at OCTranspo-A new dimension.

Washington Metropolitan Area Transit Authority. 1991. An evaluation of automated data collection systems and their use in conducting an economic route analysis.

Washington MetropolitanArea TransitAuthority.(nd).APC economic feasibility.

\section{About the Authors}

Michael R. Baltes and Joel R. Rey are Senior Research Associates at the Center for Urban Transportation Research (CUTR) at the University of South Florida in Tampa. 